Tohoku J. Exp. Med., 2011, 224, 195-199

\title{
Combined Imaging with Multi-Detector Row Computed Tomography and Diffusion-Weighted Imaging in the Detection of Pancreatic Cancer
}

\author{
Ouki Yasui ${ }^{1}$ and Makoto Sato ${ }^{1}$ \\ ${ }^{1}$ Department of Surgery, Ugo Hospital, Ogati, Japan
}

\begin{abstract}
Pancreatic cancer is one of the most aggressive malignant tumor types. Its prognosis is extremely poor without early detection, and an accurate diagnosis with imaging techniques is vital for any chance of long-term survival after treatment. Despite the great technical advances that have been made with various kinds of imaging equipment, detection of pancreatic cancer is unsatisfactory, and new modalities are required. Diffusion-weighted imaging (DWI) on magnetic resonance imaging (MRI) has high sensitivity and specificity for the detection of pancreatic cancer; however, it does not provide anatomic information. Quite often, the necessary spatial correlation of a high-intensity area with anatomic structures is constrained. If it becomes possible to combine the imaging technique of DWI with multi-detector row computed tomography (MDCT), such as is done with positron emission tomography (PET) and computed tomography (CT), it is expected that the early diagnostic capability will improve. The objective of this study was to introduce combined imaging with DWI and CT into clinical practice to improve the diagnosis of pancreatic cancer. In the current report, we demonstrate a clinical attempt to combine DWI on MRI with the anatomical accuracy of MDCT for two patients of pancreatic adenocarcinoma. Analysis of these two patients revealed that the combined images corresponded precisely to the operative findings. Thus, the combined imaging with DWI and MDCT is useful for the detection of the pancreatic cancer.
\end{abstract}

Keywords: combination; Diffusion-weighted imaging; fusion; multi-detector row computed tomography; pancreatic cancer

Tohoku J. Exp. Med., 2011, 224 (3), 195-199. C 2011 Tohoku University Medical Press

Pancreatic cancer is one of the most lethal human cancers, characterized by early systemic dissemination and extraordinary local tumor progression. Pancreatic cancer is characterized by a late presentation, and for all stages, the five-year survival rate is less than $5 \%$ (Gold and Goldin 1998). This poor prognosis relates to the uniformly advanced disease stage present at the time of diagnosis and its profound resistance to existing therapies. Thus, early detection of pancreatic cancer is required to improve this poor prognosis.

In recent years, advancements in imaging equipment have been remarkable, and the new modalities are considered indispensable for the diagnostic imaging of malignant tumors. Computed tomography (CT) is well established for the diagnosis and staging of malignant tumors. The introduction of multi-detector row computed tomography (MDCT) has allowed further refinements in detecting malignant tumors (McNulty et al. 2001; Fletcher et al. 2003). One of the most important advantages of MDCT scanners is their ability to generate multiplanar reformatted (MPR) images or 3-dimensional (3D) volume-rendered images. MPR images obtained with MDCT have high qual- ity, providing high resolution without slice misregistration.

Diffusion-weighted imaging (DWI) is different from morphologically oriented imaging techniques in that it has the sensitivity to depict disease-associated changes of random translational molecular motion, known as diffusion or Brownian water motion (Lyng et al. 2000). In tissue characterization, the relative assessment of the magnitude of diffusion is exhibited as the apparent diffusion coefficient (ADC) (Roth et al. 2004). In DWI, the signal intensity is inhibited in tissues with high diffusion of water molecules, and high-intensity signals can be obtained in tissues with low diffusion of water molecules. In most cases, malignant tumors have increased cell density and reduced intercellular substances, resulting in inhibited diffusion of water molecules. Thus, such tumor tissues produce abnormal signals by creating contrast with normal tissues.

MDCT provides excellent analysis of anatomical structures, while MRI provides excellent diagnostic performance of density resolution and tissue characterization; as for DWI, the description of the tumor has been improved by the recent development of new software. Improvements in the diagnostic capabilities of these general purpose

Received April 11, 2011; revision accepted for publication June 4, 2011. doi: 10.1620/tjem.224.195

Correspondence: Ouki Yasui, Ugo hospital, 44-5 Otomichi, Nishimonai, Ugo town, Akita 012-1131, Japan.

e-mail: ouki@sweet.ocn.ne.jp 
machines will be further expected from their convenience and more widespread use in the future.

Although the sensitivity and accuracy of MDCT and MRI in diagnosis of pancreatic cancer have been improved significantly, it is still difficult to diagnosis at an early stage (Bronstein et al. 2004; Kartalis et al. 2009). The sensitivities and specificities were reported as $73 \%-94 \%$ and $95 \%$ for CT (DelMaschio et al. 1991; Koito et al. 1997), 85\% and $96 \%$ for MRI (Ichikawa et al. 2001). The difficulties in diagnosis on MDCT are thought to be due to the loss of biliary dilatation, vascular involvement and mass effect, and little attenuation difference compared with normal parenchyma. On the other hand, MRI is a little inferior in analysis of anatomical structures including the pancreas.

In recent years, the digital image fusion procedure was developed, which enables spatially correct superposition of data from 2 or more different imaging modalities. To achieve better anatomic resolution, the combination with MDCT and DWI on MRI (DWI/CT), like the matched images with fluorodeoxyglucose positron emission tomography and $\mathrm{CT}$, will improve further the anatomical localization of the tumor and the determination of the histological and the qualitative contrast.

In the current report, we demonstrate the usefulness of fusion analysis of MDCT and DWI for the diagnosis of pancreatic cancer.

\section{Methods}

\section{MDCT imaging protocol}

MDCT was performed with a commercially available MDCT scanner (Aquilion 64, Toshiba Medical Systems, Tokyo, Japan) with a gantry rotation speed of $0.5 \mathrm{~s}$ and a detector configuration of $32.0 \times$ $1.0 \mathrm{~mm}$; the moving speed of the table was $27 \mathrm{~mm} / \mathrm{s}$. The pitch was 0.84. All scans were acquired in a cephalocaudal direction. Patients underwent MDCT followed by arterial, portal, and equilibrium phases. Patients received $100 \mathrm{ml}$ of iomeprol (Iomeron, $350 \mathrm{mg} / \mathrm{ml}$, Bracco-Eisai, Otsuka, Japan) with a monophasic injection technique by means of a power injector. The contrast material was administered at a rate of $3.3 \mathrm{ml} / \mathrm{s}$. Each phase acquisition was initiated with 35 seconds for the arterial phase, 70 seconds for the portal phase, and 150 seconds for the equilibrium phase after the injection of contrast material. MDCT examination was performed at $120 \mathrm{kVp}$ and 150 $\mathrm{mA}$. After MDCT examination, coronal and sagittal MPR images were also created. For reconstructed axial and MPR images, a 1.0$\mathrm{mm}$ slice thickness and a 0.5 -mm interval were used.

\section{MRI protocol}

All examinations were performed with a Gyroscan Intera 1.5 T containing a synergy body coil (Philips Medical Systems, Best and Heeren, The Netherlands). The apparatuses and sequences used in this study were purchased from the manufacturer as Release 12.6. All are commercially available products. The MRI protocol for localization of the tumor was as follows. T2-weighted (T2W) turbo spin echo MRI was carried out with a $37.5 \times 37.5-\mathrm{cm}$ field of view (FOV), an echo time (TE) of $80 \mathrm{~ms}$, and a repetition time (TR) of $630 \mathrm{~ms}$. Eight-mm-thick slices were acquired with a 1-mm gap between slices using a $256 \times 256$ matrix. The number of slices was 20 . T2W was performed using respiratory triggering.

Diffusion-weighted single-shot echo planar imaging using a sensitivity-encoding technique (SENSE-DWI) was carried out with a $37.5 \times 37.5-\mathrm{cm}$ FOV; a 5-mm slice thickness, a TR of 3,704 ms, a TE of $68 \mathrm{~ms}$, a $256 \times 256$ matrix, and the partial Fourier factor was 0.604 . The number of slices of parallel acquisition was 50. Receiver bandwidth is $1,246.9$ hertz. Scan time was 5 minutes and 57 seconds. A spectral presaturation with inverse recovery (SPIR) pulse was used for fat suppression. Data were acquired with b-value settings at zero and $800 \mathrm{~s} / \mathrm{mm}^{2}$. Seven images were acquired for each slice; the first was a T2-weighted image with $b=0$. The images of the next six with $\mathrm{b}=800$ were placed in each direction. DWI was performed using respiratory triggering.

\section{Imaging analysis}

Visual evaluation was performed on each of the above-mentioned images and on the fusion images, which were synthesized from T2-weighted (T2W), DWI, and MDCT. Each of the fusion images of T2W and DWI-I/800 images was synthesized on a workstation with Microsoft Windows XP (Microsoft Corporation, Redmond, WA, USA) and Philips Research Imaging Development Environment software for image registration (Philips Medical Systems, Best and Heeren, The Netherlands). The combined images with MDCT and DWI were analysed using a 3D workstation (Ziostation version 2.0. $\times$, Ziosoft, Tokyo, Japan), which is a commercially available automated image registration software. For this, the CT and DWI data were transferred onto the workstation as DICOM (Digital Imaging and Communications in Medicine) images via the Intranet. The CT images were registered to DWI using a fully automatic voxel-based algorithm. After registration, the coregistered images were reconstructed and visualized in the coronal and axial planes. CT, MRI, and combined images were assessed by one experienced radiologist who was blinded to patient information with regard to the detection of malignancies, possible infiltration of adjacent tissue or lymph nodes, and distant metastases.

\section{Patients}

Among patients with suspected lesions of the pancreas, we identified two patients in whom the assessment by observers unaware of the diagnosis showed that interpretation of CT and DWI separately gave fewer correct diagnoses than reading the matched image. This study was approved by the ethical commission on research on humans of Ugo Hospital before patient enrollment. Informed consent was obtained from each patient.

Patient 1: A 72-year-old male was admitted to our hospital because of abdominal fullness. Blood tests revealed slight hepatic dysfunction. MDCT revealed a somewhat low-density area at the site of the uncinate process of the pancreas, which revealed less enhancement relative to the normal peripheral pancreatic parenchyma (Fig. 1A). Dilatation of the bile duct was revealed, but choledochal or duodenal invasion was not considered. A low-density area $3.0 \mathrm{~cm}$ in size was in close proximity to the portal vein and the superior mesenteric artery (SMA). Coronal multiplanar reformatted images reconstructed from axial MDCT images showed a hypoattenuating mass with a spiculated border toward the portal vein and SMA. Direct tumor-to-vessel contact with complete obliteration of the fat plane was shown, which was suspected as tumor-to-vessel contiguity. DWI on MRI clearly revealed a high-intensity area at the site of the unci- 
A

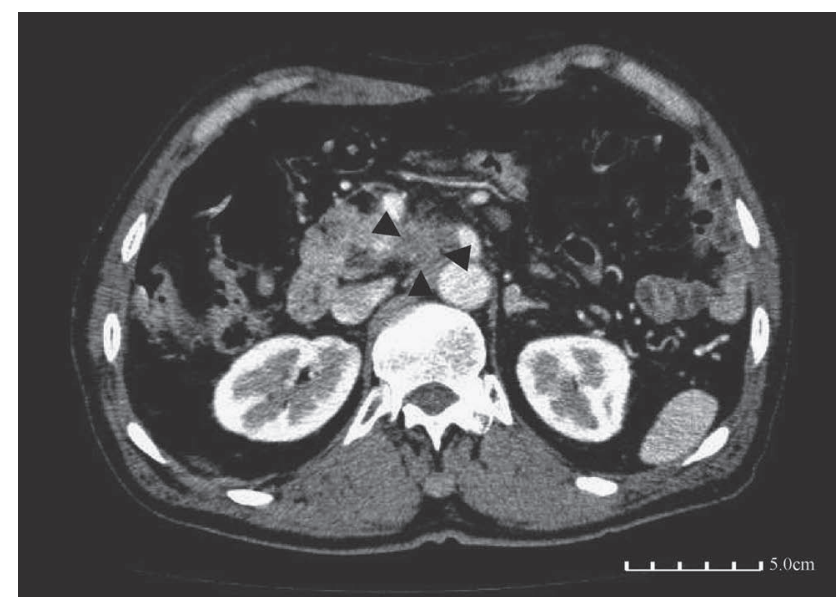

B

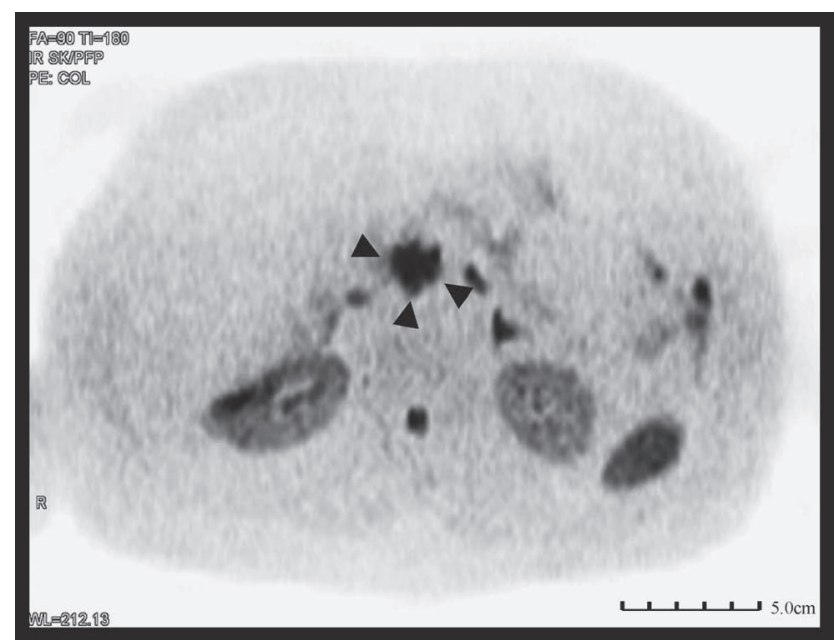

$\mathrm{C}$

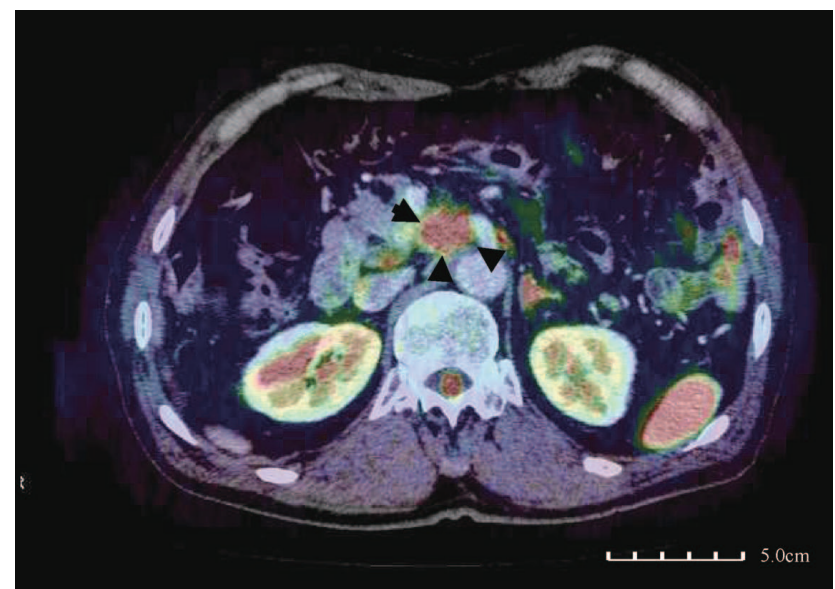

Fig. 1. Patient 1. A 72-year-old male with cancer of the uncinate process of the pancreas. A. Multi-detector row computed tomography (MDCT) images showed less enhancement relative to the normal peripheral pancreatic parenchyma at the site of uncinate process of the pancreas $(\triangleright)$. B. Greyscale inversion of diffusion-weighted imaging (DWI) showed a high-intensity area at the site of the uncinate process of the pancreas $(\nabla)$. C. Combined image with MDCT and DWI revealed both the lesser enhancement area on the MDCT and the high-intensity area of the DWI corresponded $(\triangleright)$.

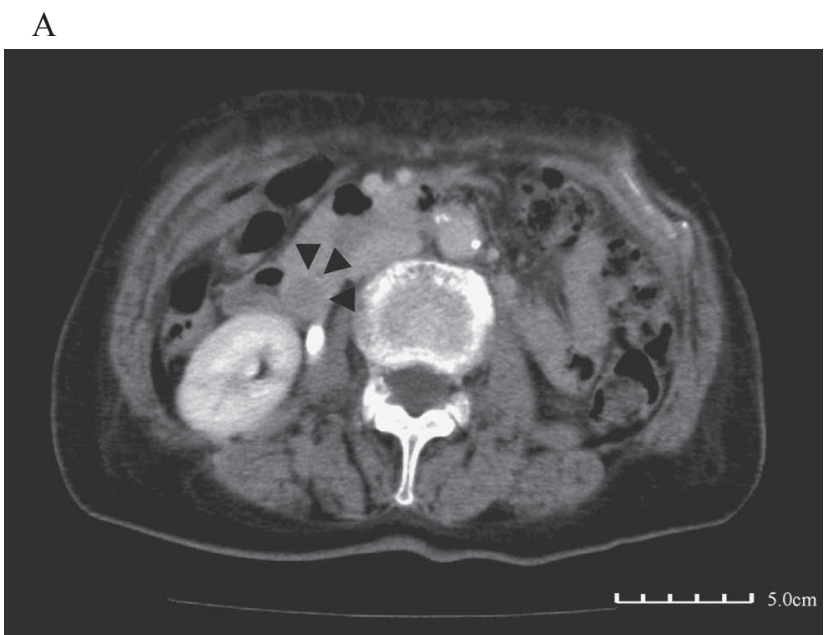

$\mathrm{B}$

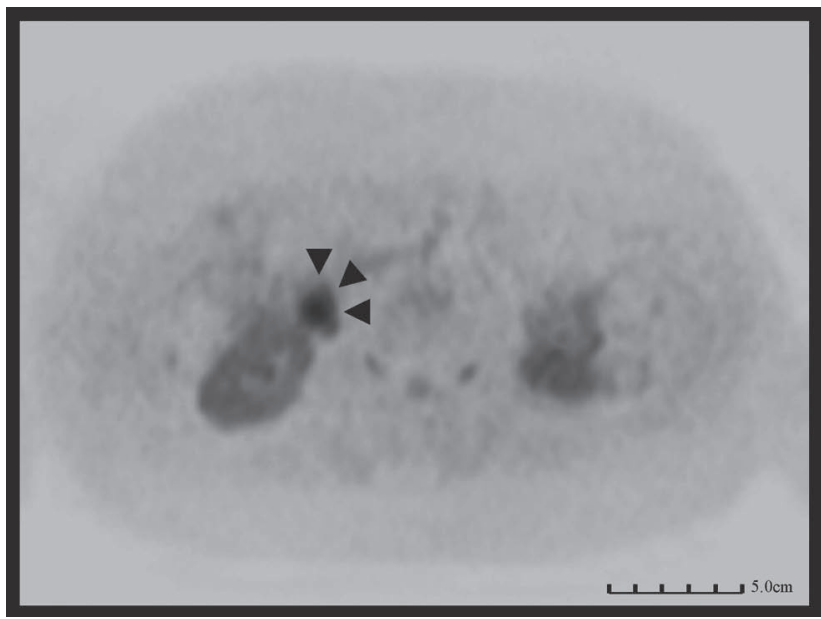

C

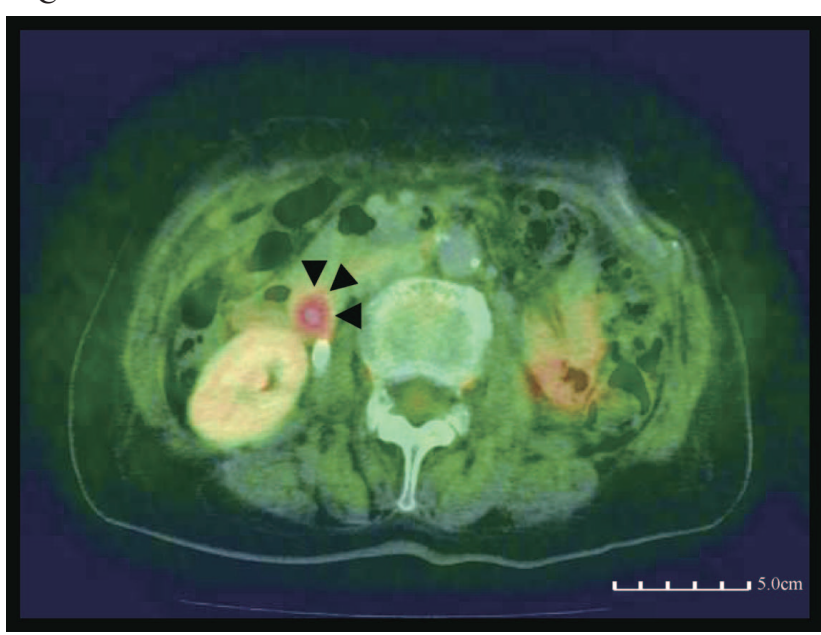

Fig. 2. Patient 2. A 76-year-old female with cancer of the head of the pancreas. A. Multi-detector row computed tomography (MDCT) images revealed a low-density area at the site of the head of the pancreas ( ). B. Greyscale inversion of diffusion-weighted imaging (DWI) showed a high-intensity area at the site of the head of the pancreas $(\triangleright)$. C. Combined image with MDCT and DWI revealed both a low-density area on the MDCT and the high-intensity area of the DWI corresponded $(\triangleright)$. 
nate process of the pancreas (Fig. 1B). The DWI and MDCT images were fused, and both the lesser enhancement area on the MDCT and the high-intensity area of the DWI corresponded (Fig. 1C). Cancer of the uncinate process of the pancreas was strongly suspected. Pancreatoduodenectomy was performed. Resected specimens revealed that the tumor was located in the uncinate process of the pancreas and was $3 \mathrm{~cm}$ in size, which corresponded to the imaging findings. Histological findings revealed moderately differentiated tubular adenocarcinoma.

Patient 2: A 76-year-old female was admitted to our hospital because of abdominal discomfort. Blood tests revealed elevation of transaminase. MDCT revealed a low-density area at the site of the head of the pancreas, $2 \mathrm{~cm}$ in size (Fig. 2A). DWI on MRI revealed a high-intensity area at the site of the head of the pancreas (Fig. 2B). The combined image with DWI and MDCT revealed that the lowdensity area on the MDCT and the high-intensity area of the DWI corresponded (Fig. 2C). Cancer of the head of the pancreas was strongly suspected. Pancreatoduodenectomy was performed. Resected specimens revealed that the tumor was located in the head of the pancreas and was $2 \mathrm{~cm}$ in size, which corresponded to the imaging findings. Histological findings revealed papillary adenocarcinoma.

\section{Discussion}

The development of various kinds of diagnostic equipment, such as sonography, CT, and MRI, has contributed to improved diagnosis and staging of pancreatic cancer; however many pancreatic cancer cases are detected at advanced stages. Because of early lymphatic and hematogenous spread and a propensity for invasive growth, only $3-22 \%$ of pancreatic cancers are resectable at the time of diagnosis (Roche at al. 2003). Several years ago, functional imaging was introduced as a new diagnostic modality in addition to conventional morphologic imaging. Functional FDG-PET has higher sensitivity and specificity than CT for tumor detection (Kantorová et al. 2003; Antoch et al. 2004). Zimny et al. (1997) reported that PET showed an overall sensitivity of $85 \%$, a specificity of $84 \%$, a negative predictive value of $71 \%$, and a positive predictive value of $93 \%$. In a subgroup of patients with normal serum glucose levels, the results were $98 \%, 84 \%, 96 \%$ and $93 \%$, respectively. Delbeke et al. (1999) demonstrated that FDG-PET had a higher sensitivity, specificity and accuracy than CT in diagnosing pancreatic cancer $(92 \%, 85 \%$ and $91 \%$ for $\mathrm{FDG}-$ PET versus $65 \%, 61 \%$ and $65 \%$ for CT, respectively). Potential roles of FDG-PET in pancreatic cancer imaging have been demonstrated, but functional imaging does not have the same resolution and landmarks as anatomic imaging. This limitation has been overcome by using co-registered functional PET and anatomic CT images (PET/CT), which has been used more commonly (Hosten et al. 2000). Lemke et al. (2004) compared the diagnostic value of CT, PET, and PET-CT fusion in 104 patients with suspected pancreatic lesions. They reported that image fusion improved the sensitivity of malignancy detection from $76.6 \%$ (CT) and $84.4 \%$ (PET) to $89.1 \%$ (image fusion).
Compared to CT alone, image fusion increased the sensitivity of detecting infiltration of adjacent tissue from $47.7 \%$ to $68.2 \%$. Furthermore, in unresectable, locally advanced pancreatic cancer (LAPC), Topkan et al. (2008) compared $\mathrm{CT}$ with co-registered PET as the basis for delineating the gross tumor volume for planning of radiation therapy. They revealed the usefulness of PET/CT-based target volume delineation in patients with LAPC, and they suggested that the potential benefit of incorporating PET into radiation therapy might be the reduction in geographic misses associated with CT-based planning.

As another imaging approach, DWI on MRI has been applied to the evaluation of pancreatic cancer. The sensitivity and specificity for the detection of pancreatic adenocarcinoma were reported as $96.2 \%$ and $98.6 \%$, respectively (Ichikawa et al. 2007). Regarding its high sensitivity and specificity, DWI has also become another imaging method for pancreatic cancer. MRI generally provides better tissue contrast than other diagnostic imaging methods, and it enables simultaneous observations of parenchymatous organs, vessels, and soft tissues. CT is considered better for the depiction of morphology, because it has superior spatial and temporal resolution. MRI is considered better for the depiction of tissue characterization, because it has superior contrast resolution. MRI is considered useful for the examination of lesions, including malignant lesions, and there are high expectations for DWI to be effective in the body trunk region. However, DWI cannot evaluate local extension of the tumor and the relationship of the tumor with the surrounding vessels. Therefore, DWI is always additional and complementary to anatomic imaging in patients considered for some treatments. Consequently, evaluations of the size and morphology of the tumor and lymph node and their internal characteristics must be performed by such combinations of methods. If fusion imaging of MDCT and DWI can be performed, it can be a useful diagnostic modality. In the present study, we used new equipment for image analysis to diagnose pancreatic cancer. The disadvantage of $\mathrm{DWI} / \mathrm{CT}$ is that it cannot be performed simultaneously with the patient in the same body posture, unlike PET/CT. As a result, it will be necessary to develop a fusion method for organs affected by peristalsis, such as the intestines. Fusion of PET and CT images is an ideal combination, but PET/CT is available only at a limited number of facilities at the present time. Other issues include exposure to radioactive isotopes and high cost. Fusion of DW and CT images can be performed at any facility with MRI and $\mathrm{CT}$, which are more common.

DWI/CT might become a useful modality for diagnosis and treatment if the resolution and image quality of DWI can be improved, the detectability of lesions can be improved, findings can be accumulated, and the development of image analysis software can be advanced. 


\section{Conflict of Interest}

The authors have no conflict of interest.

\section{References}

Antoch, G., Saoudi, N., Kuehl, H., Dahmen, G., Mueller, S.P., Beyer, T., Bockisch, A., Debatin, J.F. \& Freudenberg, L.S. (2004) Accuracy of whole-body dual-modality fluorine-18-2fluoro-2-deoxy-D-glucose positron emission tomography and computed tomography (FDG-PET/CT) for tumor staging in solid tumors: comparison with CT and PET. J. Clin. Oncol., 22, 4357-4368.

Bronstein, Y.L., Loyer, E.M., Kaur, H., Choi, H., David, C., DuBrow, R.A., Broemeling, L.D., Cleary, K.R. \& Charnsangavej, C. (2004) Detection of small pancreatic tumors with multiphasic helical CT. Am. J. Roentgenol., 182, 619-623.

Delbeke, D., Rose, D.M., Chapman, W.C., Pinson, C.W., Wright, J.K., Beauchamp, R.D., Shyr, Y. \& Leach, S.D. (1999) Optimal interpretation of FDG PET in the diagnosis, staging and management of pancreatic carcinoma. J. Nucl. Med., 40, 1784-1791.

DelMaschio, A., Vanzulli, A., Sironi, S., Castrucci, M., Mellone, R., Staudacher, C., Carlucci, M., Zerbi, A., Parolini, D. \& Faravellim, A. (1991) Pancreatic cancer versus chronic pancreatitis: diagnosis with CA 19-9 assessment, US, CT, and CT-guided fine-needle biopsy. Radiology, 178, 95-99.

Fletcher, J.G., Wiersema, M.J., Farrell, M.A., Fidler, J.L., Burgart, L.J., Koyama, T., Johnson, C.D., Stephens, D.H., Ward, E.M. \& Harmsen, W.S. (2003) Pancreatic malignancy: value of arterial, pancreatic, and hepatic phase imaging with multidetector row CT. Raiology, 229, 81-90.

Gold, E.B. \& Goldin, S.B. (1998) Epidemiology of and risk factors for pancreatic cancer. Surg. Oncol. Clin. N. Am., 7, 67-91.

Hosten, N., Lemke, A.J., Wiedenmann, B., Böhmig, M. \& Rosewicz, S. (2000) Combined imaging techniques for pancreatic cancer. Lancet, 356, 909-910.

Ichikawa, T., Sou, H., Araki, T., Arbab, A.S., Yoshikawa, T., Ishigame, K., Haradome, H. \& Hachiya, J. (2001) Duct-penetrating sign at MRCP: usefulness for differentiating inflammatory pancreatic mass from pancreatic carcinomas. Radiology, 221, 107-116

Ichikawa, T., Erturk, S.M., Motosugi, U., Sou, H., Iino, H., Araki, T. \& Fujii, H. (2007) High-b value diffusion-weighted MRI for detecting pancreatic adenocarcinoma: preliminary results.
Am. J. Roentgenol., 188, 409-414.

Kantorová, I., Lipská, L., Bêlohlávek, O., Visokai, V., Trubaĉ, M. \& Schneiderová, M. (2003) Routine (18)F-FDG PET preoperative staging of colorectal cancer: comparison with conventional staging and its impact on treatment decision making. $J$. Nucl. Med., 44, 1784-1788.

Kartalis, N., Lindholm, T.L., Aspelin, P., Permert, J. \& Albiin, N. (2009) Diffusion-weighted magnetic resonance imaging of pancreas tumours. Eur. Radiol., 19, 1981-1990.

Koito, K., Namieno, T., Nagakawa, T. \& Morita, K. (1997) Inflammatory pancreatic masses: differentiation from ductal carcinomas with contrast-enhanced sonography using carbon dioxide microbubbles. Am. J. Roentgenol., 169, 1263-1267.

Lemke, A.J., Niehues, S.M., Hosten, N., Amthauer, H., Boehmig, M., Stroszczynski, C., Rohlfing, T., Rosewicz, S. \& Felix, R. (2004) Retrospective digital image fusion of multidetector CT and $18 \mathrm{~F}-F D G$ PET: clinical value in pancreatic lesions-a prospective study with 104 patients. J. Nucl. Med., 45, 12791286.

Lyng, H., Haraldseth, O. \& Rofstad, E.K. (2000) Measurement of cell density and necrotic fraction in human melanoma xenografts by diffusion weighted magnetic resonance imaging. Magn. Reson. Med., 43, 828-836.

McNulty, N.J., Francis, I.R., Platt, J.F., Cohan, R.H., Korobkin, M. \& Gebremariam, A. (2001) Multi-detector row helical CT of the pancreas: effect of contrast-enhanced multiphasic imaging on enhancement of the pancreas, peripancreatic vasculature, and pancreatic adenocarcinoma. Radiology, 220, 97-102.

Roche, C.J., Hughes, M.L., Garvey, C.J., Campbell, F., White, D.A., Jones, L. \& Neoptolemos, J.P. (2003) CT and pathologic assessment of prospective nodal staging in patients with ductal adenocarcinoma of the head of the pancreas. Am. J. Roentgenol., 180, 475-480.

Roth, Y., Tichler, T., Kostenich, G., Ruiz-Cabello, J., Maier, S.E., Cohen, J.S., Orenstein, A. \& Mardor, Y. (2004) High-b-value diffusion-weighted MR imaging for pretreatment prediction and early monitoring of tumor response to therapy in mice. Radiology, 232, 685-692.

Topkan, E., Yavuz, A.A., Aydin, M., Onal, C., Yapar, F. \& Yavuz, M.N. (2008) Comparison of CT and PET-CT based planning of radiation therapy in locally advanced pancreatic carcinoma. J. Exp. Clin. Cancer Res., 27, 41.

Zimny, M., Bares, R., Fass, J., Adam, G., Cremerius, U., Dohmen, B., Klever, P., Sabri, O., Schumpelick, V. \& Buell, U. (1997) Fluorine-18 fluorodeoxyglucose positron emission tomography in the differential diagnosis of pancreatic carcinoma: a report of 106 cases. Eur. J. Nucl. Med., 24, 678-682. 